\title{
Philosophiques
}

Marco Panza et Jean-Michel Salanskis (éds.), L'objectivité mathématique. Platonismes et structures formelles, Paris, Masson, 1995, X-241 p.

\section{Yvon Gauthier}

Volume 24, numéro 1, printemps 1997

Avez-vous lu Rawls?

URI : https://id.erudit.org/iderudit/027443ar

DOI : https://doi.org/10.7202/027443ar

Aller au sommaire du numéro

Éditeur(s)

Société de philosophie du Québec

ISSN

0316-2923 (imprimé)

1492-1391 (numérique)

Découvrir la revue

Citer ce compte rendu

Gauthier, Y. (1997). Compte rendu de [Marco Panza et Jean-Michel Salanskis (éds.), L'objectivité mathématique. Platonismes et structures formelles, Paris, Masson, 1995, X-241 p.] Philosophiques, 24(1), 217-220.

https://doi.org/10.7202/027443ar

Ce document est protégé par la loi sur le droit d'auteur. L'utilisation des services d'Érudit (y compris la reproduction) est assujettie à sa politique d'utilisation que vous pouvez consulter en ligne.

https://apropos.erudit.org/fr/usagers/politique-dutilisation/ 
Marco Panza et Jean-Michel Salanskis (éds.), L'objectivité mathématique. Platonismes et structures formelles, Paris, Masson, 1995, $\mathrm{X}-241 \mathrm{p}$.

Ce recueil de textes veut mettre en lumière le platonisme ou les platonismes que certains voient encore comme une option possible de la philosophie des mathématiques. Un autre nom pour ce platonisme-là serait sans doute structuralisme et des auteurs américains comme Resnick et Shapiro utilisent le terme au sens où je l'ai introduit dans un article de 1974 ("Constructivisme et structuralisme dans les fondements des mathématiques ". Philosophiques (1) p. 83-105). Comme les fondements sans fondationnalisme de $\mathrm{S}$. Shapiro (voir 
son Foundations without Foundationalism : A Case for Second-Order Logic, Oxford. Oxford University Press, 1991), il faudrait peut-être parler de structures sans structuralisme ou de Platon sans platonisme, si l'on voulait appuyer un tant soit peu.

Le premier article de l'historien des mathématiques, Jean Dhombres, sur - Un platonisme spontané : le témoignage contradictoire des textes mathématiques "évoque deux questions, les racines imaginaires de Descartes et la continuité au sens de Cauchy. Pour la première, il rappelle que Kronecker a produit une théorie constructive de domaines de rationalité qui incorpore les racines imaginaires dans un corps commutatif dès 1882 (Dhombres cite le résultat de 1887) et pour l'autre il s'interroge à bon droit sur la pertinence de l'analyse informelle de Lakatos. Un platoniste pourrait cependant rétorquer eu égard à la première question que Steinitz a donné en 1910 (Algebraische Theorie der Körper) une extension transcendante de la théorie kroneckerienne en admettant des méthodes de preuve non constructives, e.g. l'axiome du choix.

Dans son texte "Arguments et indices dans le débat sur le réalisme mathématique *. J.-P. Delahaye dresse un bilan prudent du réalisme en présentant une hiérarchie à quatre paliers de questions mathématiques à portée ontologique, de la théorie des nombres et de la combinatoire finie à la théorie des ensembles transfinis. L'auteur conclut, après un exposé très pédagogique, qu'on peut trouver de bonnes raisons de monter les premières marches, mais qu'on doit s'arrêter probablement à l'hypothèse du continu, l'infini dénombrable étant suffisant pour l'appétit constructiviste, ou prédicativiste à la Feferman. L'auteur, quant à lui, attend en spectateur intéressé de voir ce qui va se passer en mathématiques et en logique pour prendre une décision ontologique en faveur du réalisme.

C'est un peu l'attitude de J.-M. Salanskis dans son article " Platonisme et philosophie des mathématiques", mais le recul que procure l'herméneutique philosophique lui permet d'espérer une * analyse existentiale de l'Ėtre des objets de la mathématique " (p. 212). Alain Badiou dans "Platon et/ou AristoteLeibniz. Théorie des ensembles et théorie des Topos sous l'œil du philosophe * s'engage résolument sur la voie transcendantale en déclarant qu'il n'existe pas, ni ne peut exister de philosophie des mathématiques et cependant que la théorie des ensembles contraint à des options philosophiques de type platonicien et que la théorie des catégories et des topoi contraint à des options philosophiques de type aristotélicien, ou leibnizien. Ainsi le "pullback " - l'auteur ne le traduit pas, mais il s'agit du produit fibré - montrerait le primat du virtuel sur l'actuel, rapport qui serait inversé dans la théorie des ensembles où le produit direct serait sans doute plus direct, si l'on suit le raisonnement analogique de l'A. L'A. omet évidemment de noter que la notion de topos, née en géométrie algébrique, est une généralisation de la notion d'espace topologique dans l'esprit de Grothendieck et qu'elle a un rôle fondationnel " analogue " à celui de la théorie des ensembles dans l'utopie des U-topoi ou univers de Grothendieck! Estil besoin de rappeler que les ouverts d'un espace topologique obéissent à une logique intuitionniste, tout comme un topos arbitraire?

Jean Petitot emboite le pas dans cet élan philosophique et son texte * Pour un platonisme transcendantal " est une revue de la littérature en théorie des ensembles contemporaine et reproduit à la lettre des résultats dont l'A. offre un commentaire littéral. Le constat final de ce platonisme qui est transcendantal s'appuie sur l'objectivité d'une notion de continu, corrélat de l'intuition suprasensible qu'a invoquée Gödel et qui parait "philosophiquement parfaitement justifiée •(p. 178), aux yeux de l'A. On mesurera la pertinence des remarques 
philosophiques de l'A. à propos du principe de réflexion dans la théorie axiomatique des ensembles de Zermelo-Fraenkel avec axiome du choix : "Cette réflexion de $\mathrm{M}$ (modèle de $\mathrm{Z}-\mathrm{F}-\mathrm{C}$ ) dans un de ses propres modèles intérieurs est philosophiquement très profonde*(p. 176). Or il est facile de montrer que le principe de réflexion est équivalent à l'axiome de remplacement plus l'axiome de l'infini dans Z-F. (voir Y. Gauthier, Fondements des mathématiques, P.U.M., 1976. p. 40-41). En d'autres mots, ce qui est logiquement manifeste est philosophiquement profond (en apparence !). En réalité, on n'est pas très loin ici d'une Penelope Maddy dont la demière version du réalisme s'appelle naturaliste et qui consiste essentiellement à justifier de facto la propension naturelle des praticiens de la théorie des ensembles à " croire aux axiomes ", quel qu'en soit le coût fuduciaire.

Une attitude plus critique est défendue par $P$. Engel dans son article "Platonisme mathématique et antiréalisme " et il se rapproche là-dessus de J.P. Delahaye. D'une discussion éclairée des thèses de Dummett, C. Wright et $\mathrm{H}$. Field, Engel ne tire pas cependant les éléments d'une solution personnelle, préfèrant sans doute l'ambivalence de Frege à la fin de sa vie : "Parfois j'en vois une, parfois je n'en vois pas "à une question de Wittgenstein à propos des difficultês de sa théorie du nombre - notons en passant que Wittgenstein est à peu près absent de ce recueil ; son antiplatonisme eùt ètè pourtant un utile contrepoids (voir là-dessus l'ouvrage de M. Marion, Wittgenstein, Finitism, and the Foundations of Mathematics, à paraītre chez Clarendon Press, Oxford, 1997). Poincaré. dont l'antilogicisme était aussi antiplatoniste, y eūt ajouté encore plus de poids.

Dans la mème veine, le texte de Jacques Harthong ( " L'idéalisme platonicien et la science ") est davantage un plaidoyer informel pour le constructivisme entendu au sens large qu'une critique radicale du platonisme cantorien. L'A. plaide pour un platonisme naif qu'on pourrait opposer au platonisme transcendantal d'un Petitot. C'est plutôt de ce côté que se range "Platonisme et intentionnalité " de $\mathrm{M}$. Panza dans une réflexion philosophique qui se situe dans le prolongement des textes de Petitot et Salankis. Les observations d'ensemble de la fin du volume dues à M. Panza et J.-M. Salankis et qu'on pourrait lire comme un résumé récapitulatif du recueil reprennent d'ailleurs le thème d'une sorte de dialectique œcuménique qui montre comment les platonismes sont accordés entre eux, si tant est qu'il existe un dénominateur commun aux engagements ontologiques en deça ou au-delà des engagements fondationnels. La distinction est importante. puisque le volume ne comporte aucun texte qui relève d'une pratique fondationnelle concrète - seul l'article de J. Harthong pouvant être considèré comme l'êcho du travail de G. Reeb en arithmétique des entiers naifs. On peut d'ailleurs renvoyer à un autre recueil du mème genre (Le Labyrinthe du continu, édité par J.-M. Salankis et H. Sinaceur, Paris, SpringerVerlag, 1992) dont le seul texte qui témoigne d'une pratique fondationnelle est celui d'E. Nelson - il faut relever ici une faute d'impression importante en page 158 : " $P$ is inconsistent " et non " $G$ is inconsistent ", puisque $P$ est l'arithmétique de Peano et $G$ l'arithmétique de $R$. Robinson dont une variante est autoconsistante par la preuve de Nelson dans Predicative Arithmetic (Mathematical Notes 33, Princeton University Press, Princeton, 1986).

En conclusion, malgré des textes qui sont en général bien documentés - la bibliographie est à ce titre représentative de la littérature contemporaine - on trouvera dans ce recueil davantage une illustration d'orientations platonisantes en philosophie des mathématiques que la défense philosophico-logico-mathéma- 
tique d'un point de vue fondationnel qui rende compte de l'attitude critique propre à la recherche fondationnelle.

Yvon Gauthier

Département de philosophie

Université de Montréal 